\title{
Impacts of infrastructures on reliability of urban rail bound public transport networks
}

\author{
S. Tahmasseby \& R. van Nes \\ Transport and Planning, Faculty of Civil Engineering and Geosciences, \\ Delft University of Technology, The Netherlands
}

\begin{abstract}
For the analysis of public transport networks, it is assumed that the network performs as planned. In reality, however, there are many disturbances influencing infrastructure networks and public transport services. In other words disturbances cause service quality variations and thus affect service reliability. These variations are more critical in rail bound public transport networks due to the fact that they need exclusive infrastructures for operation. In this article, we look at a public transport network design methodology that accounts for reliability and robustness of rail bound urban public transport service networks by considering impacts of stochastic events. The study shows the role of infrastructures such as bypasses, shortcuts and turning facilities to improve public transport network robustness. We apply the methodology in a real case study for the tram network: city of The Hague. Results show a sensible improvement in network connectivity reliability as well as total network costs.
\end{abstract}

Keywords: reliability, public transport networks, infrastructures, robustness.

\section{Introduction}

Operating rail bound public transport networks requires exclusive infrastructures. However, infrastructures might not always be available as planned. A number of events influence the availability of public transport infrastructures. For instance, it is possible that some parts of an infrastructure are blocked for a while due to events such as traffic accidents or a broken-down tram. In these situations, the services cannot continue working. If suitable infrastructure alternatives are available, the public transport operator may continue the affected lines' operation and thus maintain service network connectivity for instance, by using detours or 
splitting the service line into two parts. For rail-bound services this requires special attention. In other words, for rail bound public transport networks detours and $U$ turns at terminals are only possible using specific infrastructures. Hence, considering service network connectivity in public transport network design leads to a new infrastructure network design problem. A possible design question is to determine whether and where additional infrastructures such as bypasses, shortcuts and turning infrastructures should be provided in a public transport infrastructure network. Furthermore, the network design problem could be extended by including a reliability term into traditional network design objective functions. This issue is addressed in this article by analyzing the impacts of adding extra infrastructures to a tram network. We consider and apply different types of infrastructure: the bypass track, the shortcut track and turning track. These infrastructures are tested in a case study separately and corresponding results in terms of network robustness and cost are assessed. For implementation we choose a real case study; The Hague tram network. We first determine vulnerable points in the network by determining connectivity reliability and then given the assessment we look for adding the indicated infrastructures to improve the network performance. The assessment criteria are network connectivity reliability, travel costs, and total network costs. Our approach clearly shows that including infrastructure facilities in a tram network will increase network connectivity reliability and even might lead to a considerable reduction in travel costs and perhaps in total network costs.

\section{Disturbances on public transport networks}

An implicit requirement in public transport network evaluation studies is that the necessary infrastructure is available with appropriate quality. In reality, however, this is not always the case. Public transport services often share infrastructure with other modes, while in the case of the dedicated infrastructure there are still many crossings with other traffic that suffer from congestion and incidents. Furthermore, infrastructure needs maintenance, and since it is part of the city it might be affected by other building or maintenance activities for e.g. sewers, cables, et cetera. Basically, track, signal (including safety and communication systems), power supply, and planned construction works are some main causes for infrastructure faults (Veiseth et al. [10]). Also, the impact of external events should not be forgotten. Bad weather and road works are typical external events that may happen in any public transport network and cause disturbances.

Moreover, some events might be correlated in time or space. Local circumstances influence incidents (Schreuder et al. [6]). For instance, the probability of incidents may increase in bad weather conditions (Edwards [3]). This relationship is not straightforward; the weather influences the frequency of road accidents by affecting both the volume of traffic, and therefore the number of road users exposed to risk, and the risk per unit of travel (Codling [2]). Another example is the correlation between bad weather and road works. The probability of road works increases substantially after extreme weather conditions such as snow or storm due to damaged infrastructures. Also, the 
probability of incidents is higher at locations such as bridges or tunnels that suffer from bad weather consequences.

\section{Stochasticity in public transport networks}

Given these events which may affect infrastructure availability, the question is how these events might influence the public transport services. Basically, there are two types of effects:

- Part of the services is out of order (e.g. the scheduled services is limited to a shorter path or might even be split into two different parts, depending on the location of disturbances).

- Detours are implemented. Transport services will be maintained by diverting the route using the available infrastructure.

In general a public transport service provider will try to maintain the services as good as possible. As such, it is likely that they will attempt to provide detours. Only in the case that no detours can be provided, services will be skipped from the schedule, for instance in the case of multiple disturbances along a line, or in the case of specific weather conditions.

Applying detours can affect the operational costs for public transport operators. Detours require additional driving time and thus additional operational costs. The actual impact, however, might be limited if the extra time is less than the scheduled buffer time for the public transport service involved. Anyway, applying any adjustment such as detour needs essential infrastructures which their availability determines robustness of a network.

Furthermore, travellers who are confronted with unexpected changes in public transport service supply usually have limited possibilities to change their behaviour. In the case of detours they will have to accept the additional travel time. Also, they should make decision en-route and look for alternatives routes or even they are forced to postpone their trips. Thus, extra travel and perhaps trip cancelation costs are imposed to the indicated travellers.

\section{Connectivity reliability in public transport networks}

In order to measure the robustness of a public transport network, we define connectivity reliability as "the probability that the network nodes remain connected". On the supply side, it can be measured as the probability that network nodes are still connected (Al-Deek and Emam [1]). The network is successful if the existing lines are in operation as planned. In case of disruptions in the system, the probability of service line failures affect the connectivity reliability on the supply side. For instance, it can be quantified as the number of service runs that could not reach their destinations.

On the demand side, connectivity reliability in public transport networks might for example be quantified as the number of trips that could not reach their destination.

Meanwhile, it would be of interest to consider public transport modes by type of right of way in this analysis. Public transport networks with the highly 
protected right of way such as Metro lines are less sensitive to external events such as incidents, bad weather and public events; however, operational adjustments are quite difficult as well due to required dedicated infrastructures. Adversely, public transport networks with shared right of way such as bus networks suffer more from external events; however, operational adjustments are much easier as well. For instance, a bus driver can divert the bus from original route via a detour to avoid a road blockage and keep the service in operation while for a tram driver applying a detour is not easy since dedicated infrastructures are needed. In the latter case, detours are only possible using the infrastructures that are already used by other rail-bound public transport services or using additional shortcuts and turnings in the rail infrastructure.

With respect to connectivity reliability, having the infrastructure available for shortcuts and turnings in a public transport network might be a fruitful option to prevent service disruptions and increase network robustness. A typical example is the availability of a ring infrastructure in a radial service network. An infrastructure shortcut might be used as a backup in case of emergency to provide detour facilities for an operator. For further information please refer to (Tahmasseby and van Nes [7]).

\section{Including reliability in public transport network design}

Basically, the main challenge in the traditional public transport network design is to determine a network with an optimal performance given a specific design objective and possible constraints. Normally in order to address the viewpoints of the traveller and the investor or the operator; public transport network designers choose a composed network design objective function which balances different objectives. Fan and Machemehl [4] and van Nes [8] use minimising total network costs as a network design objective function. It minimises the sum of costs involved in travelling, that is the total door-to-door travel time monetised using the value of time, plus the operating cost, investments cost, and maintenance cost. Formula 1 shows the objective of minimising total network costs mathematically:

$$
\operatorname{Min}\left(C_{t}+O C+I C\right)
$$

Where:

$C_{t}$ : travel costs

$O C: \quad$ service operation costs

$I C: \quad$ infrastructure investment and maintenance costs

The optimisation problem above might also include an operational budget constraint. In the aforementioned formula, all network costs components are assumed to be constant over time. However, this is not a realistic assumption as indicated already. To cope with stochasticity, we modify the design objection function to be more realistic. We consider travel time variations. We also include network connectivity reliability in the objective function by means of trip cancellation penalty. Furthermore, extra travel time imposed to travellers as well as extra operation costs imposed to the operator due to possible detours. Thus, we define a public transport network design objective function in the stochastic 
perspective. Formula (2) shows the network design objective function including reliability on an aggregate level:

Where:

$$
\operatorname{Min}\left[\left(C_{t}^{*}+O C+I C\right)+\left(C_{t e}+C_{t c}\right)+\left(O C_{e}\right)+\left(I C_{e}\right)\right]
$$

$C_{t}^{*}$ : travel costs including regular travel time variations

$C_{t e}$ : extra travel costs in non-recurrent conditions

$C_{t c}: \quad$ trip cancellation cost in non-recurrent conditions

$O C_{e}$ : extra operation costs in non-recurrent conditions

$I C_{e}$ : extra investment costs for building infrastructure shortcut possibilities for detours

Note that extra travel time in a non-recurrent condition is imposed to the travellers when they are forced to travel via longer routes or detours, transfer between lines, or travel a part of their route on foot.

\section{Case study}

To assess the impacts of infrastructures on network reliability, a model has been developed to assess the impacts of various kinds of stochastic events on performance of public transport networks. The model captures the operation adjusting scenarios as well as travellers' behaviour in case of disturbances in the network discussed in section 3 .

As the application an existing public transport network in the city of The Hague in the Netherlands has been analyzed. The tram network (see figure 1) consists of 48 centroids with mainly a predefined radial infrastructure. The tram network has a high percentage exclusive right of way $(85 \%)$. The tram operator, HTM, operates 10 tram lines using 67 links with a total length of $135 \mathrm{~km}$. The public transport demand pattern is known by travellers' surveys (HTM [5]). About 140 million trips are made in a year for the public transport network. Public transport demand is primarily centre oriented, while some sub centres (e.g. the cities of Delft, Rijswijk, Voorburg and Leidschendam) also attract their share of the demand.

The network is analyzed for 20 different simulation runs representing 20 different years, in which events take place. A Monte-Carlo approach is used to select failing links, while for simplicity sake no correlation between failing links is assumed. The frequency and duration of each event type is based on realistic assumptions. For the interval between two events with the same type, a negative exponential distribution is applied, while the duration is modelled using a lognormal distribution. Bad weather including storm, black ice, heavy snow, as well as public events, road works, incidents, and vehicle breakdown are examples of such events. The parameters for frequency and duration of each type of event are determined based on realistic estimates (e.g. Dutch climate). The events are sorted along the time axis, so that for each moment in time it is known whether there is a disturbance and if so, which type(s). Please note that multiple events might take place at the same time. Also, the correlation between events is considered. For further details, please see references (Van Nes et al. [9]; Tahmasseby and van Nes [7]). 


\subsection{Scenarios}

We first evaluate the Hague tram network in a stochastic perspective, compare to deterministic perspective and then we assess the impact of extra infrastructures on network robustness and the network design objective function. Hence, we design our scenarios as follows:

1. Reference network (2008), as shown in figure 1. This network is analyzed in two ways: the deterministic and the stochastic perspective.

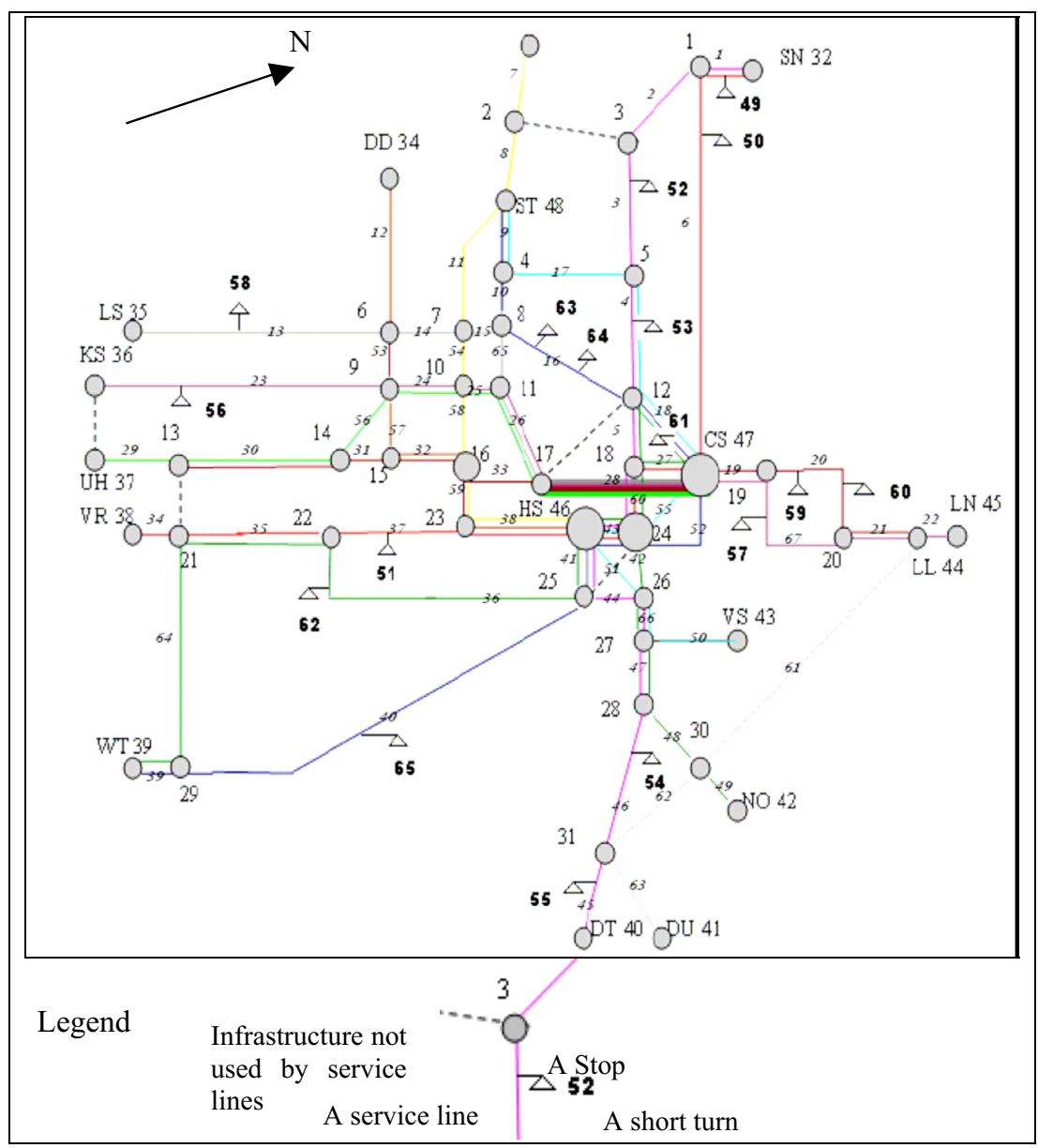

Figure 1: Tram Network of the Hague (reference network).

2. Network with a bypass near Central Station (CS). The link between Central Station and the northern part of the network has proven to be vulnerable. This link is shown in figure 2-A (link number 19). 
3. Network with a shortcut connecting terminal Loosduinen (LS 35) of line 3 to terminal Krayensteinlaan (KS 36) of line 2. This shortcut is shown figure 2-B.

4. Network with a turning facility at station Scholekstersingel (30) which allows turning from node 28 to 31 and vice versa (figure 2-C).

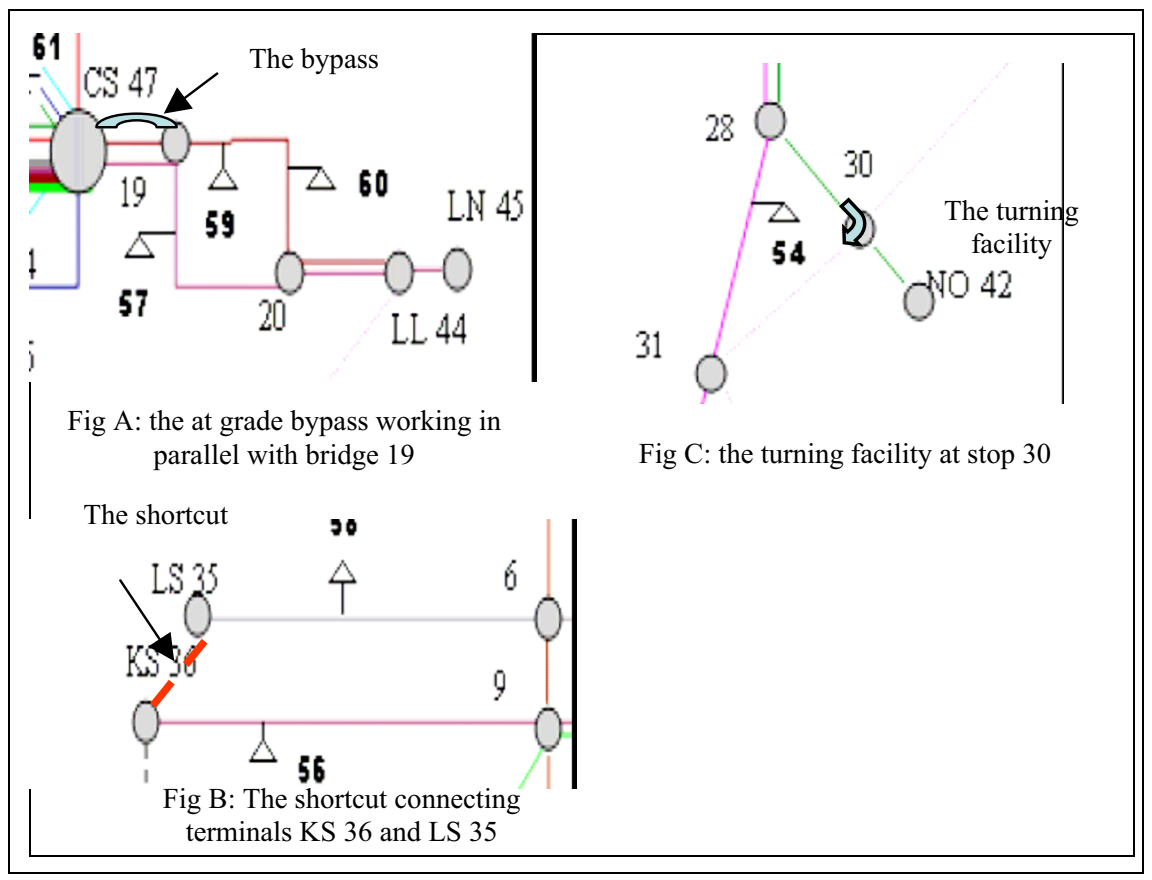

Figure 2: Details of additional infrastructures applied in the reference network.

\subsection{Results}

This part discusses obtained results for the cases described in the previous part. Figure 3 shows the relative difference in link load per link in the reference network. It shows that some links are used up to $23 \%$ more in the non recurrent situation (e.g. link 9\&10) that means these links are used as detours when other links are blocked. Other links are used less in the non recurrent case that means these links have no function as a bypass. Probably no good bypasses exist; hence, if these links are blocked, trips will be cancelled and link loads will decrease. These results are very useful as a start to assess the robustness of a public transport network.

The robustness of the Hague network is determined by extracting trip cancelation rates per origin. The results show that terminal Delft Tanthof is the most vulnerable one. The trip cancelation rate is the highest for this origin. Table 1 outlines the ten most vulnerable points in terms of connectivity 
reliability. It clearly shows that they are terminal points located at the end of service lines and do not have any alternatives when the corresponding main lines are affected.

Relative links' loads (non recurrent situations to recurrent situation)

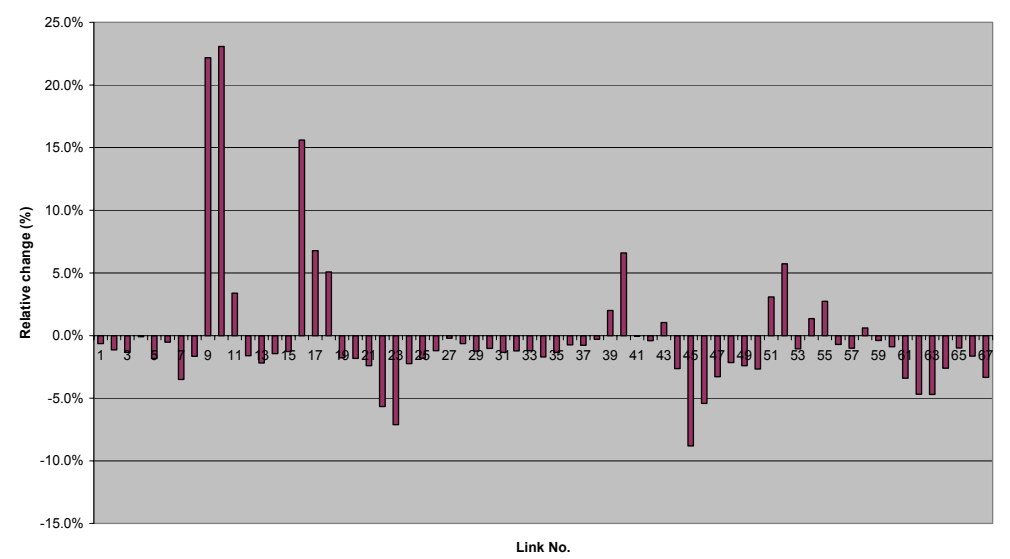

Figure 3: Relative changes in link loads (stochastic vs. deterministic)).

Table 1: Robustness of the Hague tram network: The most vulnerable origins.

\begin{tabular}{|c|c|c|}
\hline $\begin{array}{c}\text { Origin } \\
\text { number }\end{array}$ & Origin name & $\begin{array}{c}\text { The annual percentage of trip } \\
\text { cancelation (mean value) }\end{array}$ \\
\hline 40 & Delft Tanthof & $6.9 \%$ \\
\hline 43 & Voorburg Station & $5.8 \%$ \\
\hline 41 & Delft University & $5.2 \%$ \\
\hline 45 & Leidschendam North & $4.7 \%$ \\
\hline 31 & Delft Station & $4.6 \%$ \\
\hline 34 & Duindorp & $4.1 \%$ \\
\hline 44 & Leidschendam Leidschenhage & $3.5 \%$ \\
\hline 36 & Kraaijenstein & $3.0 \%$ \\
\hline 35 & Loosduinen & $2.9 \%$ \\
\hline 38 & Vrederust & $2.8 \%$ \\
\hline
\end{tabular}

As the $2^{\text {nd }}$ scenario, a bypass parallel to link 19 is added to the link 19 between Central Station and the northern part of the network (figure 2-A). The results already showed that nodes 44 (Leidschendam Leidschenhage) and 45 (Leidschendam North) suffer from high trip cancelation rate (table 1). Moreover, the indicated link is a vulnerable link and if it is blocked, there won't be any alternative for it and thus, the connection between north and south part of the city will be cut. The extra link reduces disturbances on the aforementioned terminals and corresponding lines using the link. Even, when link 19 fails, all lines using this link can still operate via the new at grade bypass. This creates benefits for travellers on other parts on the lines and network as well. The model 
demonstrates that adding the bypass can reduce number of trip cancelation even up to $24 \%$ for some years with a mean value of $9 \%$. Also, it decreases extra travel costs up to $18 \%$ with a mean value of $4 \%$. This is due to higher number of direct services providing by these robust lines. Meanwhile, because of less trip cancellations, usage of detours in the southern part of the network decreases accordingly which leads to reductions in load of some southern links (e.g. 9\&11) and city centre (e.g.16-18).

In the $3^{\text {rd }}$ scenario we connect terminals Loosduinen (LS 35) and Kraayensteinlaan (KS36) to each other by a shortcut infrastructure (figure 2-B). Note that this shortcut is a single track since it is not heavily used like the bypass and thus construction cost is lower than the bypass. It is used in case of disturbances at links 13 or 23 . Thus, the corresponding lines can be diverted from the original path and use another track, serve travellers and terminate at their original terminal via the shortcut. We have chosen these terminals because annual percentages of trip cancelation at them are relatively high (table1). Results show there is a $2.4 \%$ reduction in trip cancelation on average. This rate reaches up to $11.9 \%$ for some years; however, extra travel costs increases about $1.52 \%$ due to the fact more trips are made via this shortcut.

In the $4^{\text {th }}$ scenario we facilitate node 30 with a right turn possibility (figure 2-C). Thus, if either link 45 or link 46 is blocked in line 1, the line could continue operation via a diversion through links 48 and 62 . This detour can be only provided if an extra facility for turning exists at node 30 . The results already showed that terminal Delft Tanthof DT40 suffers from the highest trip cancelation rate (table 1). Also, line 1 is a major line in the city because it connects west side of The Hague to east side of Delft. Hence, applying this turning possibility will increase network robustness. The model proves that adding the turning facility can reduce number of trip cancelation even up to $22.9 \%$ for some simulated years with a mean value of $6.2 \%$. Also, it decreases extra travel costs down to $8.9 \%$ with a mean value of $2.6 \%$. This is due to higher number of direct services providing by line 1 in the city centre, and suburbs. This reduction results in a $0.35 \%$ decrease in total network costs.

The results are more or less similar for different types of infrastructure. In other words it proves that it's possible to gain good results in terms of reliability even with small investment (bypass vs. shortcut). So, choosing the relevant location for extra infrastructures plays an important role.

\section{Conclusions}

In this article the extension of public transport network design problem is addressed with a stochastic perspective. We showed that including transport service reliability requires adjusted design objectives including new components in the formulation. Also, in a stochastic perspective when disruptions may influence the network, the links' load alters due to detours.

Furthermore, facilitating public transport networks with infrastructure shortcuts introduces a new aspect of strategic network design, especially for rail bound public transport networks. Our approach clearly shows that introducing 
facilities such as infrastructure shortcuts will increase network connectivity reliability. The analyses for the Hague tram network prove that additional infrastructures can not only increase the robustness of the network, but also they could lead to a significant reduction in travel costs and even in total network costs. Obviously the methodology introduced in this paper could be elaborated further. Selecting the best locations and the best investment for applying extra infrastructures such as shortcuts, short turn facilities, and bypasses via the optimisation, and changing service lines configuration (e.g. splitting them into two parts) and assessing effects are examples of research extensions that lead toward a reliable public transport network design.

\section{Acknowledgements}

This study is supported by the Transport Research Centre Delft and by the BSIKprogramme Next Generation Infrastructures.

\section{References}

[1] Al-Deek, H. \& Emam, E.B., 2006. New Methodology for Estimating Reliability in Transportation Networks with Degraded Link Capacities. Journal of Intelligent Transportation Systems, 10(3), p. 117-129.

[2] Codling, J.P., 1974. Weather and Road Accidents. In Climatic Resources and Economic Activity, David \& Charles, Newton Abbot, p. 205-222.

[3] Edwards, J.B., The temporal distribution of road accidents in adverse weather, Meteorological Applications 6, 59-68, 1999

[4] Fan, W., Machmehel, R.B., 2006. Using a Simulated Annealing Algorithm to Solve the Public Transport Route Network Design Problem., Journal of Transportation Engineering, 132, p.122.

[5] HTM, Department of Research and Development, 2004-2005, Public Transport OD Research Haaglanden, The Hague (in Dutch)

[6] Schreuder, M.A., Molenkamp, L., Tamminga, G.F. \& Kraan, M.E., 2007. Vulnerability of a National Road Work. The 3rd International Symposium on Transportation Network Reliability, 19-20 July 2007, The Hague.

[7] Tahmasseby, S. \& van Nes, R., 2007. Public Transport Network Design and Reliability. In: Proceedings: The 3rd International Symposium on Transportation Network Reliability, 19-20 July 2007, The Hague.

[8] Van Nes, R., 2002. Design of Multimodal Transport Networks, a Hierarchical Approach. Trail Thesis Series T2002/5, Trail Research School, Delft University Press, Delft.

[9] Van Nes, R., Tahmasseby, S. \& de Jong, P., 2006. Robustness of Transport Service Networks, Effects of Infrastructure Availability. Proceedings of the 9th TRAIL Congress - TRAIL in Motion - CD-ROM, November 2006, Rotterdam.

[10] Veiseth, M., Olsson, N. \& Saetermo, I.A.F., 2007, Infrastructure's Influence on Rail Punctuality, Proceedings of Urban Transport XIII: Urban Transport and the Environment in the $21^{\text {st }}$ Century. September 2007, Coimbra. 